\title{
DESCRIPCIÓN DE UNA ESPECIE NUEVA DE MELANIPS WALKER (HYMENOPTERA: FIGITIDAE), PRIMER REGISTRO DEL GÉNERO PARA LA REGIÓN NEOTROPICAL
}

\author{
JULI PUJADE-VILLAR ${ }^{1}$ \& JUAN M. VANEGAS-RICO ${ }^{2}$ \\ ${ }^{1}$ Universitat de Barcelona, Facultat de Biología, Departamento de Biología Animal, Avda. Diagonal 645, \\ 08028-Barcelona (España). < jpujade@ub.edu> \\ ${ }^{2}$ Programa de Entomología, Instituto de Fitosanidad, Colegio de Postgraduados, 56230 Montecillo, Texcoco, \\ Estado de México (México). < hymenopter@yahoo.com> \\ Recibido: 13/12/2013; aceptado: 22/09/2014
}

Pujade-Villar, J. \& Vanegas-Rico, J. M. 2015. Descripción de una especie nueva de Melanips Walker (Hymenoptera: Figitidae), primer registro del género para la región neotropical. Acta Zoológica Mexicana (n. s.), 31(1): 48-54.

RESUMEN. Se describe una nueva especie del género Melanips Walker (Hymenoptera: Figitidae) de México, Melanips lomeyva sp. nov. la cual representa el primer registro del género Melanips para México y para la región Neotropical. Se ilustran y discuten los caracteres diagnósticos de la nueva especie. Los especímenes estudiados se obtuvieron de pupas del díptero Leucopis bellula (Chamaemyiidae), enemigo natural de la cochinilla silvestre del nopal Dactylopius opuntiae (Dactylopiidae). Se presentan datos del huésped y de la biología de esta nueva especie.

Palabras clave: Cynipoidea, Chamaemyiidae, parasitoides, México.

\section{INTRODUCCIÓN}

Las especies de la familia Cynipidae, y otras de Figitidae, configuran el grupo de los microcinipoideos que producen agallas, principalmente en encinos (Ronquist 1999). Los Figitidae en México son poco conocidos, pues sólo se conocen cerca de 60 especies (Pujade-Villar, datos no publicados), de las 1500 estimadas que integran a la familia (Ronquist 1999, Buffington et al. 2005). Algunos grupos de figítidos son parasitoides primarios de dípteros (Diptera: Schizophora), presentes en hábitats como minas de hojas, excrementos y carroña (Ronquist 1999, Buffington 2002, Fontal-Cazalla et al. 2002). Las subfamilias Eucoilinae y Figitinae pueden parasitar a estos dípteros (Weld 1952, Ronquist 1999, Ros-Farré et al. 2000, Buffington 2002), además de la subfamilia Aspicerinae, en la cual se ubica actualmente el género Melanips.

Melanips es un género controvertido, que anteriormente estaba incluido en la subfamilia Figitinae, pero tras un análisis genético se transfirió a la subfamilia Aspicerinae (Buffington et al. 2007). No obstante, la ausencia de las dos sinapomorfias presentes en ese grupo (Ros-Farré et al. 2000), tales como la impresión facial y la forma en "silla de montar" del segundo segmento metasomal, podría motivar inconsistencia morfológica motivaría su regreso a Figitinae (Paretas-Martínez et al. 2011a). Me-
Pujade-Villar, J. \& Vanegas-Rico, J. M. 2015. Description of a new species of Melanips Walker (Hymenoptera: Figitidae), first record of the genus for the Neotropical region. Acta Zoológica Mexicana (n. s.), 31(1): 48-54.

ABSTRACT. A new species of Melanips Walker (Hymenoptera: Figitidae) from Mexico, Melanips lomeyva sp. nov. is described. This species represents the first record of the genus Melanips from Mexico and to the Neotropical region. Diagnostic characters of the new species are illustrated and discussed. The adults were obtained from pupae of Leucopis bellula (Chamaemyiidae) natural enemy of wild cochineal of prickly pear Dactylopius opuntiae (Dactylopiidae). Biological data and hosts of this new species are commented.

Key words: Cynipoidea, Chamaemyiidae, parasitoids, Mexico.

lanips podría formar una subfamilia independiente dentro de Figitidae junto con otros géneros recientemente descritos morfológicamente similares (Nebulovena Pujade-Villar \& Paretas-Martínez 2011 y Ferpereira Pujade-Villar 2012). Las seis especies de Melanips descritas para el continente americano presentan una distribución Neártica, y de estas, ninguna se cita para México.

Además de las controversias taxonómicas, existen muy pocos datos referentes a la biología de Melanips. Algunas especies atacan larvas de dípteros de la familia Syrphidae y Chamaemyiidae (Gilreath \& Smith 1988, Ferguson 1986). Por lo tanto, el presente estudio describe una nueva especie de Melanips, incluyendo información sobre su biología.

\section{MATERIAL Y MÉTODOS}

El muestreo se efectuó en huertas comerciales de nopal verdura Opuntia ficus-indica (L.) Miller, ubicadas en el municipio de Tlalnepantla (Estado de Morelos) y en la delegación Milpa Alta (Distrito Federal). Las dos demarcaciones suman el 76\% de la producción de "nopalitos" en México (SIAP 2013). Otros muestreos se realizaron en forma esporádica en los municipios Nezahualcóyotl, Nicolás Romero y Texcoco (Estado de México), Juchitepec 
(Morelos) y Espinal (Veracruz). Dichos sitios, al igual que muchos otros de la República Mexicana, incorporan a $O$. ficus-indica en pequeños huertos de traspatio o como un elemento de ornato.

La colecta del material entomológico se realizó de acuerdo con el método descrito por Vanegas-Rico et al. (2010), para la evaluación de la cochinilla silvestre del nopal Dactylopius opuntiae (Cockerell) (Hemiptera: Dactylopiidae). Se realizaron muestreos semanales desde febrero 2008 a febrero 2009, cada uno de los cuales incluyó 50 colonias maduras (hembras en producción de huevos) de $D$. opuntiae, sobre las cuales se presentan sus depredadores. Cada colonia se colocó individualmente en una caja Petri de $2.5 \mathrm{~cm}$ de diámetro, y se trasportó al laboratorio de control biológico del Colegio de Postgraduados (Texcoco, México). Bajo un estéreo-microscopio, se contó y registró el número de insectos entomófagos presentes, colocando las larvas en cajas Petri y alimentándolas con colonias de $D$. opuntiae criadas en laboratorio. Las pupas se mantuvieron individualmente en cápsulas de gelatina dura número "0". Los depredadores y sus parasitoides emergidos se etiquetaron, algunos de ellos se preservaron en viales (tipo Eppendorf) con alcohol 70\% para estudios posteriores, mientras que otros se montaron en seco en triángulos de cartulina blanca. El material estudiado se depositó en la colección de insectos del Colegio de Postgraduados, Montecillo (CPM, México) y en la colección privada (JP-V) resguardada en Universitat de Barcelona (UB, Catalunya).

La nomenclatura taxonómica se basó en los trabajos de Liljeblad \& Ronquist (1998), Melika (2006) y Ros-Farré \& Pujade-Villar (2006, 2007), siguiendo la terminología de Harris (1979). Las imágenes se tomaron en los "Serveis Cinetífico - Tècnics de la Universitat de Barcelona” con el microscopio electrónico de barrido Stereoscan S-360 (Cambridge Instruments); éstas fueron obtenidas a bajo voltaje (1000 V) en el caso de los machos sin cubrimiento de oro y a alto voltaje (10 KV) después de haber cubierto las muestras en oro. Las imágenes del habitus y del primer par de alas se tomaron con una cámara PAXcam $3^{\text {tm }}$ integrada (Midwest Information System) acoplada a un estereo-microscopio Tessovar (Zeiss, Alemania), en el laboratorio de Morfología de Insectos del Colegio de Postgraduados.

Melanips lomeyva Pujade-Villar y Vanegas-Rico, sp. nov.

(Figs. 1-3)

Material tipo. HOLOTIPO $q$ (JPV-UB): MEXICO. “MEX: DF, Tepenahuac, Del. Milpa Alta (19¹1'17”N, 9859’36”O) 2,460 msnm, J.M. Vanegas-Rico col.” [etiqueta blanca], "Ex: Leucopis bellula Williston, 1889 (Chamaemyiidae), en Dactylopius spp. (Hemiptera: Dac- tylopiidae), en Opuntia ficus-indica (L.) 09-v-2013" [etiqueta blanca]. "Holotipo + Melanips lomeyva PujadeVillar \& Vanegas-Rico n. sp., JP-V desig. 2013” [etiqueta roja]. PARATIPOS MEXICO: con los mismos datos del holotipo; "MEX, Edo. México, Nicolás Romero, Col. Zapata (19³6’25” N, 99¹6’42”O) 2,383 msnm, 02-v-2009 J.M. Vanegas-Rico col." (1ठ̂); “MEX, Edo. México Texcoco, Universidad Autónoma Chapingo (19²9’34”N, 9853’04”O) 2,261 msnm, 02-viii-2008, J.M. Vanegas-Rico col." (3); "MEX, Edo. México, Juchitepec (1906’29”N, 9853'03”O) 2,537 msnm, 26-x-2008, J.M. Vanegas-Rico col." (2+); "MEX, DF., Milpa Alta, Calle Veracruz (19¹0’49”N, 9859'24”O) 2,554 msnm, 07vii-2010, J.M. Vanegas-Rico col.” (2 9 ); “MEX, Morelos, Tlalnepantla, Parcela Baja (1859’00”N, 9859’11”O) 1,741 msnm, 30-x-2007, J.M. Vanegas-Rico col." (10๊, 1 ㅇ); idem 27-ii-2009 (2今); "MEX, Morelos, Tlalnepantla, Parcela Eleazar (1901’04”N, 9859'30”'O) 2,132 msnm, 12-ix-2008, J.M. Vanegas-Rico col." (20); “MEX, Morelos, Tlalnepantla, Parcela Esteban (1900’07’N, 9900’47”O), 2,200 msnm, 12-x-2008, J.M. VanegasRico col." (19); "MEX, Morelos, Tlalnepantla, Parcela Juan (1859’35”N, 9859'39”O) 1,849 msnm, 28-iv-2008 J.M. Vanegas-Rico col.” (2犬, 1 ㅇ); Parcela Vigía (Tlalnepantla, Morelos) (1900’22”N, 9858’10”O), 2,105

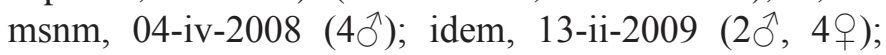
“MEX, Morelos, Tlalnepantla, Aguapilzitla (1901’31”N, 9859’46”O), 2,332 msnm, 02-vii-2012, J.M. VanegasRico col." (1 $\left.\odot, 6{ }^{\Uparrow}\right)$; "MEX, Veracruz, Espinal, Entabladero (20¹5’28”N, 97²4’15”O), 100 msnm, 11.v.2011: Nuvia Orduño-Cruz col” (1우).

Diagnosis. Antenas con los segmentos del flagelómero subiguales, alrededor de 2 veces más largos que anchos; F1 en el macho curvado, excavado y ensanchado en la parte distal. Notaulos incompletos. Surco medio ausente. Líneas parapsidales y líneas paralelas anteriores presentes. Carenas del propodeo delimitando una zona romboidal. Celda radial cerrada, 2.3 veces tan larga como ancha. Segundo terguito metasomal provisto de con dos placas densamente pubescentes. Tercer terguito metasomal con una fina banda punteada cerca del margen posterior y siguientes terguitos ampliamente punteados.

Descripción. Longitud total del cuerpo: Hembras: 2.2-2.3 mm; machos, 2.0-2.1 mm.

Coloración. Cabeza y mesosoma completamente negros; metasoma negro, castaño oscuro a negro en los segmentos finales e hipopigio. Antenas castañas, escapo y pedicelo oscuros. Patas castañas, coxas negras en los 2/3 apicales y fémures oscurecidos en el centro. Alas translucidas, venación castaño claro (Fig. 1a).

Cabeza. En vista frontal 1.1-1.2 veces más ancha que alta con pubescencia blanquecina corta, uniformemente dis- 

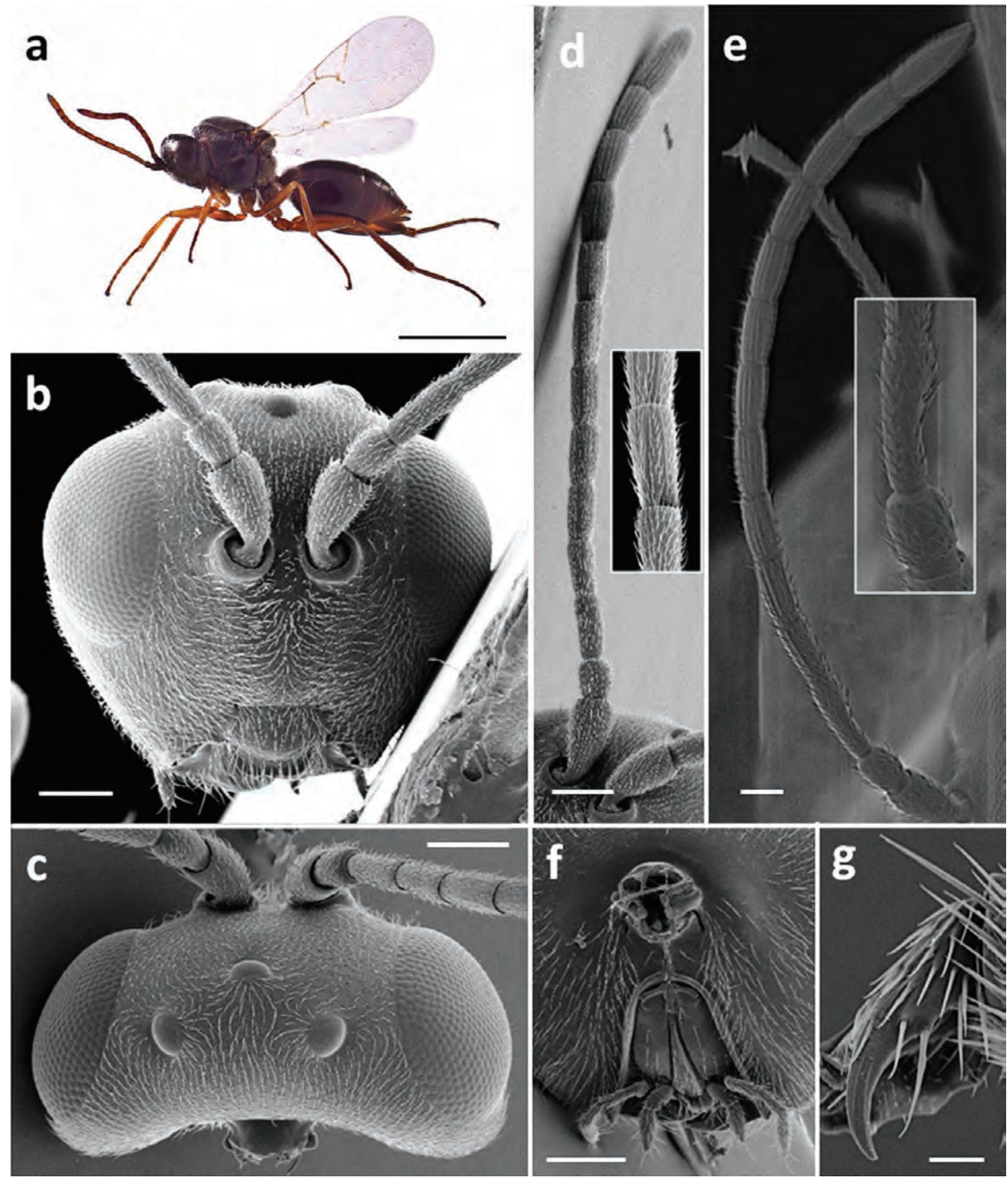

Figura 1. Melanips lomeyva sp. nov. : (a) habitus; (b) cabeza en vista frontal; (c) cabeza en vista dorsal; (d) antena de la hembra y detalle del F2; (e) antena del macho y detalle del F1; (f) área inferior de cabeza en vista posterior; (g) uña tarsal. Escalas: a = 1 mm, b-f = $100 \mu$ m, g = $10 \mu \mathrm{m}$.

tribuida. Escultura coriácea. Espacio malar 0.6 veces la altura del ojo compuesto. Líneas clípeo-pleurostomales y surco epistomal delimitando el clípeo, fosetas tentoriales presentes, aunque pequeñas. Clípeo esculpido, margen ventral proyectado medialmente por encima de las man- díbulas, lobulado. Línea transfacial ligeramente mayor que la altura del ojo compuesto (11:10). Diámetro de los toruli ligeramente superior a la distancia entre el toruli y el ojo compuesto; distancia entre los toruli igual a 1/3 de su diámetro (Fig. 1 b). Ocelos laterales ligeramente por 

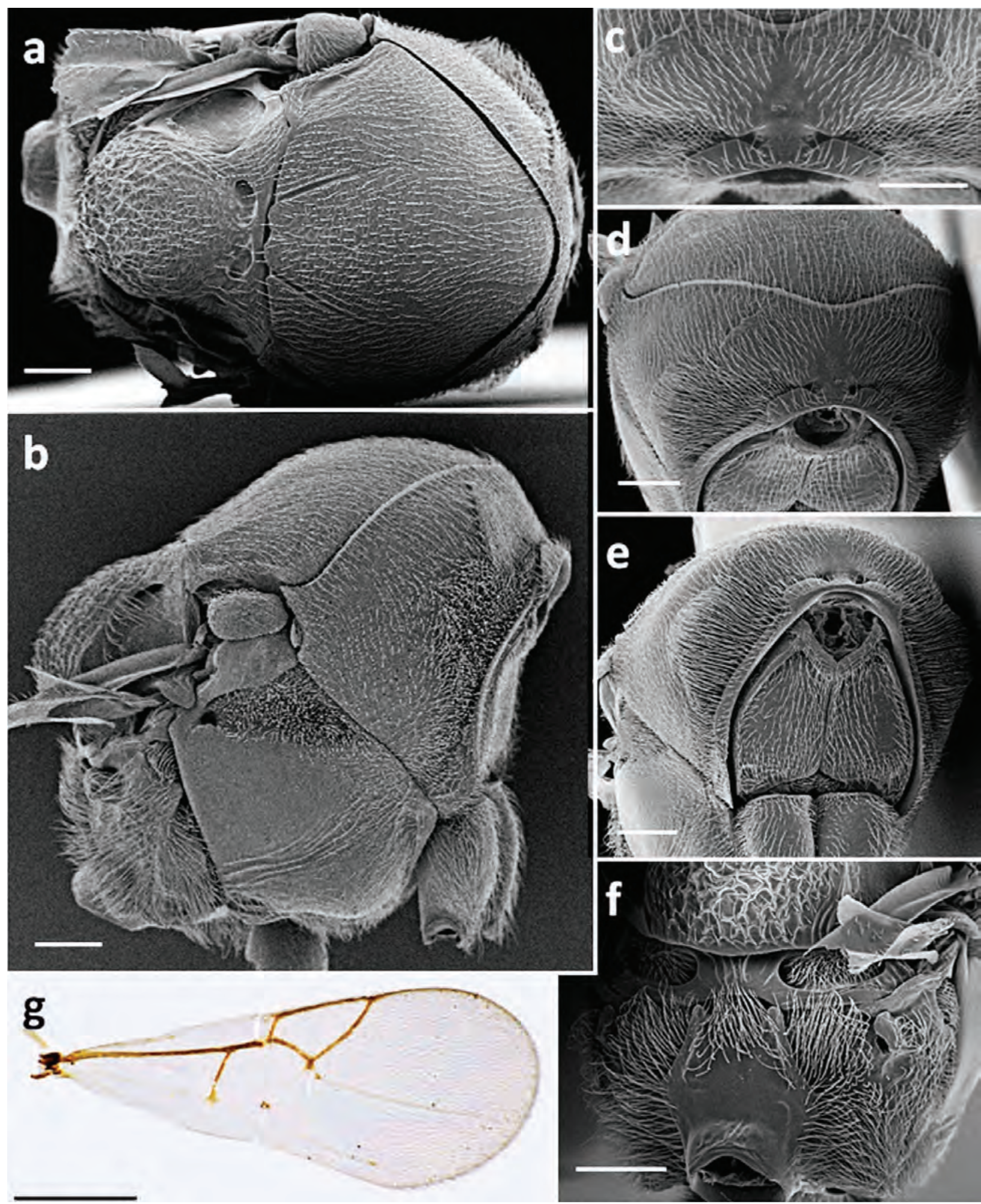

Figura 2. Melanips lomeyva sp. nov.: (a) mesosoma en vista dorsal; (b) mesosoma en vista lateral; (c) detalle del propodeo en vista dorsal; (d) mesosoma en vista frontal; (f) propodeo; (g) primer par de alas. Escalas: a-f $=100 \mu \mathrm{m}, \mathrm{g}=1 \mathrm{~mm}$.

encima del margen lateral de la cabeza. En visión dorsal, alrededor de 2 veces tan ancha como larga (21:10), igualmente pubescente que la cara y ligeramente más ancha que el mesosoma (21:20). Escultura coriácea. POL es casi
2 veces OOL; OCO ligeramente mayor que el diámetro del ocelo lateral; la relación POL: OOL: OCO es 5:2.5:2, siendo el diámetro del ocelo lateral igual a 1.75 (Fig. 1e). Antenas. Hembras con 13 segmentos, de los cuales F1 
es ligeramente más largo y estrecho que F2; F2-F10 subiguales en longitud; F2-F3 ligeramente más estrechos; sensilias presentes a partir de F2, aunque escasas y menos conspicuas en este segmento y situadas en la parte dorsal; F11 ligeramente inferior a el doble de la longitud del F11 (22:13) (Fig. 1d). Macho con 14 segmentos; F1-F11 subiguales, dos veces más largos que anchos; F1 ligeramente curvado, excavado y ensanchado distalmente; sensilias presentes a partir de F3; F12 ligeramente inferior al doble de la longitud del F11 (5.5:3) (Fig. 1e).

Mesosoma. Ligeramente más estrecho que la cabeza, claramente más largo que ancho (27:20) (Fig. 2a). Pronoto coriáceo, cubierto por una pubescencia blanquecina, corta y uniformemente distribuida, más densa y larga lateral y externamente que la placa pronotal (Fig. 2 a-e). Carenas pronotales presentes, incompletas en la parte dorsal; placa pronotal no definida dorsalmente, ni proyectada. Fosetas pronotales circulares separadas por una distancia igual al doble de su diámetro (Fig. 2 d). Mesoescudo dos veces más largo que el escutelo, coriáceo, cubierto por una pubescencia blanquecina, corta y uniformemente distribuida (Fig. 2a-b). Líneas paralelas anteriores presentes indicadas por la ausencia de pubescencia; notaulos incompletos, alcanzando prácticamente la mitad del escudo superando la altura de las tégulas; líneas parapsiales presentes alcanzando el mismo nivel que lo notaulos; surco medio ausente. Escutelo ligeramente más largo que ancho, redondeado distalmente, fuertemente coriáceo, lateral y basalmente rugoso; fosetas escutelares ovales, bien definidas, poco profundas, separadas por una área menor a la anchura de la foseta (Fig. 2a). Lateralmente más largo que alto (29:25). Mesopleura longitudinalmente carenada, más fuerte en el tercio inferior; especulum liso (Fig. 2a.) Triangulo mesopleural completamente pubescente. Axila lisa, débilmente pubescente en la mitad superior. Surco mesopleural por encima de 1/3 de la altura de la mesopleura. Metaescutelo rectangular, poco inciso, basalmente, provisto de carenas longitudinales. Foseta metanotal pubescente. Propodeo fuertemente pubescente (Fig. 2b), parte inferior delimitada por las carena propodeales, liso y glabro; carenas del propodeo delimitando una zona romboidal; primero divergentes, provistas de pubescencia y luego convergentes y glabras, no definidas internamente formando una placa con el área central (Fig. 2 f).

Alas. Primer par de alas traslúcidas, más cortas que la longitud del cuerpo (66:73). Pubescentes en la superficie y en el margen. Celda radial cerrada alrededor de 2.3 veces más larga que ancha (Fig. 2g).

Patas. Uñas tarsales simples (Fig. 1g).

Metasoma. Ligeramente más largo que la cabeza y el mesosoma juntos (37:33) (Fig. 1a) y claramente más largo que alto en las hembras (Fig. 3a). Segundo terguito liso, con dos placas densamente pubescentes situadas en la zona dorsolateral anterior (Fig. 3d). Tercer terguito metasomal glabro, con una fina banda punteada cerca del margen posterior en la zona dorsolateral (Fig. 3a-c). Siguientes terguitos glabros y punteados en una amplia banda central. Hipopigio no prolongado en espina, basalmente con líneas de setas (Fig. 3e).

Etimología. En honor a los doctores J. Refugio Lomeli-Flores y Esteban Rodríguez-Leyva (Colegio de Postgraduados) por la confianza y el apoyo constante para el segundo autor, tanto moral e intelectual como financiero, en la línea de investigación sobre insectos de nopal del Programa de Posgrado en el Colegio de Postgraduados.

Distribución. México: Región Neártica: Estado de México, Distrito Federal y Morelos. Región Neotropical: Veracruz.

Huésped. Obtenido de pupas de Leucopis bellula Williston 1889 (Diptera: Chamaemyiidae), depredador de cochinillas de nopal Dactylopius spp. (Hemiptera: Dactylopiidae) sobre nopal verdura Opuntia ficus-indica (L.) Miller.

Biología. Melanips lomeyva sp. nov., es un endoparasitoide coinobionte que ataca larvas de $L$. bellula y emerge de las pupas de su hospedero. Bajo las condiciones de los cultivos de "nopal verdura" en Tlalnepantla (Morelos), las poblaciones de sus insectos asociados (cochinilla, depredadores y parasitoides), registraron la mayor abundancia entre abril y mayo, periodos con condiciones climáticas favorables para las cochinillas de nopal por la baja precipitación pluvial (con registros de siete eventos menores a $5 \mathrm{~mm}$ cada uno) y temperaturas altas ( $\mathrm{T}$. máx semanal = $26.1 \pm 0.4^{\circ} \mathrm{C}$ ). Por su parte, en la delegación de Milpa Alta, Distrito Federal se presenta un clima menos cálido, en donde se observó un pico de abundancia de $M$. lomeyva sp. nov., coincidente en tiempo y magnitud al registrado en Tlalnepantla, Morelos. En los demás sitios donde se recolectó este parasitoide corresponden a plantas de ornato (Nezahualcóyotl y Nicolás Romero, Edo México), pequeños huertos de traspatio (Juchitepec, Edo. México), además de cultivos de nopal tunero (Nopaltepec y Teotihuacan, Edo México) y en nopales silvestres que crecen a pie de carretera (El Espinal, Veracruz).

\section{DISCUSIÓN}

El género Melanips incluye poco más de 20 especies válidas (JP-V, datos no publicados), y presenta una distribución Holártica y Oriental, con la exclusión de las especies australianas, M. hartigi Girault 1930 y M. pilosiscutum Girault 1929, trasferidas a los géneros Mikeius Buffington, 2008 y Cicatrix Paretas-Martínez, 2011 (Figitidae: Mikeiinae), respectivamente (Paretas-Martínez et al. 2011b). 

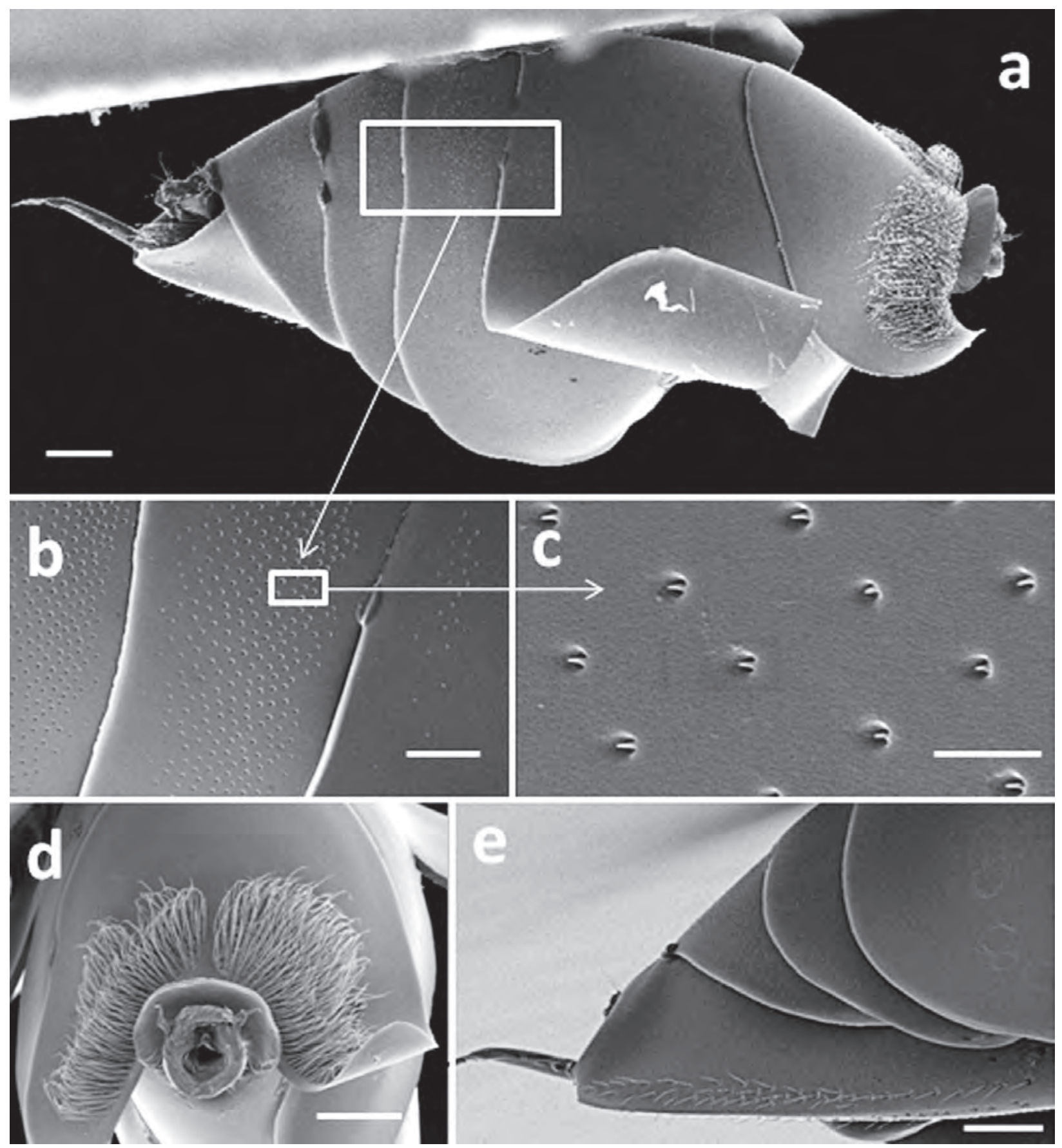

Figura 3. Melanips lomeyva sp. nov.: (a) metasoma en vista lateral; (b-c) detalle de la puntuación metasomal; (d) metasoma en vista frontal; (e) hipopigio. Escalas: a-b y d-e $=100 \mu \mathrm{m}, \mathrm{c}=10 \mu \mathrm{m}$.

Las seis especies descritas en el género Melanips corresponden a Estados Unidos de América, que se ubica en la región Neártica (Krombein et al, 1979): Melanips bilineatus (Kieffer 1909) de los estados de Idaho y Washington; M. coxalis (Kieffer 1907) de Nevada y California, incluyendo la variedad dipterorum (Kieffer 1910) de Arkansas; M. iowensis Ashmead, 1887 de Iawa; M. opacus (Hartig 1840) de California; M. semirugosus (Crawford 1917) de New York; y M. slossonae (Crawford 1917) de
New Hampshire. Tras la revisión de sus descripciones originales, se concluye que $M$. lomeyva sp. nov. es una especie válida, ya que presenta las siguientes características distintivas: tamaño del cuerpo menor a $4 \mathrm{~mm}$, notaulos incompletos (a diferencia de $M$. bilineatus, $M$. coxalis, $M$. opacus y M. slossonae), metasoma punctuado (en contraste con $M$. coxalis y $M$. iowensis por; de $M$. semirugosus), longitud del F1 muy corto (5 veces más largo que ancho en $M$. semirugosus), carinas del propodeo sin una 
curvatura pronunciada y segundo terguito metasomal sin puntuación completa (a diferencia de $M$. semirugosus); además, presenta las carinas del propodeo no paralelas, escultura del escudo no finamente puntuado y ausencia de surco medio. Finalmente, el escutelo de $M$. lomeyva no presenta una rugosidad tan pronunciada en toda su superficie.

La presencia de esta nueva especie de Melanips representa la primera referencia del género para México y la región Neotropical. Estudios previos en Texas registraron la presencia de una especie no determinada de Melanips sobre L. bellula alimentándose de Dactylopius confusus (Cockerell) (Gilreath \& Smith 1988), la cual podría corresponder a $M$. lomeyva sp. nov. Las características geográficas de México y el traslado de cladodios por los propósitos de alimento y ornato contribuyen en la dispersión de la cochinilla y todos los gremios asociados. Los sitios de cultivo intensivo de $O$. ficus-indica, pudieran favorecer la presencia de este parasitoide, debido a que en Tlalnepantla, las poblaciones de este figítido representaron el $40.4 \%$ del total de parasitoides emergidos sobre $L$. bellula; mientras que en los cultivos experimentales del Colegio de Postgraduados (Texcoco, Estado de México) rebasó el 50\%. Las recolectas en todos estos sitios sugieren que $M$. lomeyva sp. nov. se distribuye ampliamente en la región Neártica y probablemente es un parasitoide específico de L. bellula.

Agradecimientos. Agradecemos muy sinceramente a Mattias Forshage (Swedish Museum of Natural History, City and Country) por cedernos el dato del número de especies de Eucoilinae (Figitidae) presentes en México. El segundo autor agradece al Consejo Nacional de Ciencia y Tecnología (CONACYT) su apoyo económico y a su comité académico por el apoyo moral y formativo para la realización de la tesis doctoral.

\section{LITERATURA CITADA}

Buffington, M. L. 2002. Description of Aegeseucoela Buffington, new name, with notes on the status of Gronotoma Förster. Proceedings of the Entomological Society of Washington, 104: 589-601.

Buffington, M. L., Burks, R. \& Mcneil, L. 2005. Advanced techniques for imaging mircrohymenoptera. American Entomology, 51: 50-54.

Buffington, M. L., Nylander, J.A.A. \& Heraty, J. 2007. The phylogeny and evolution of Figitidae (Hymenoptera: Cynipoidea). Cladistics, 23: 1-29.

Ferguson, N. D. M. 1986. Charipidae, Ibaliddae and Figitidae (HymeNOPTERA: CynipoIdEA). HANDBOOK for the identification of British insects. Royal Entomological Society of London, 8: 1-55.

Fontal-Cazalla, F., Buffington, M., Nordlander, G., Liljeblad, J.,
Ros-Farré, P., Nieves-Aldrey, J. L., Pujade-Villar, J. \& Ronquist, F. 2002. Phylogeny of the Eucolinae (Hymenoptera: Cynipoidea: Figitidae). Cladistics, 18: 154-199.

Gilreath, M. E. \& Smith Jr., J. W. 1988. Natural enemies of Dactylopius confusus (Homoptera: Dactylopiidae): Exclusion and subsequent Impact on Opuntia (Cactaceae). Environmental Entomology, 17:730-738

Harris, R. 1979. A glossary of surface sculpturing. State of California, Department of Food and Agriculture, Occasional Papers in Entomology, 28: 1-31.

Krombein, K. V., Hurd, P. D., Smith Jr., D. R. \& Burks. B. D. 1979. Catalog of Hymenoptera in America North of Mexico. Volume 1. Symphyta and Apocrita (parasitica). Smithsonian Institution Press, Washington, D.C. Vol. 1: xvi+1-1198 pp.

Liljeblad, J. \& Ronquist, F. 1998. A phylogenetic analysis of higherlevel gall wasp relationships (Hymenoptera: Cynipidae). Systematic Entomology, 23, 229-252.

Melika, G. 2006. Gall Wasps of Ukraine. Cynipidae. Vestnik zoologii, supplement 21(1-2), 1-300, 301-644.

Paretas-Martínez, J., Rakhsani, E., Fathabadi, K. \& Pujade-Villar, J. 2011a. Description of Nebulovena persa Pujade-Villar \& Paretas-Martínez gen. n. and sp. n. (Hymenoptera: Cynipoidea: Figitidae: Figitinae) from Iran, with a key to the genera of Figitinae. Zootaxa, 3177: 43-51.

Paretas-Martínez, J., Restrepo-Ortiz, C., Buffington, M. \& Pujade-Villar, J. 2011b. Systematics of Australian Thrasorinae (Hymenoptera, Cynipoidea, Figitidae) with descriptions of Mikeiinae, new subfamily, two new genera, and three new species. Zookeys, 108: 21-48.

Ronquist, F. 1999. Phylogeny, classification and evolution of the Cynipoidea. Zoologica Scripta, 28: 139-164.

Ros-Farré, P. \& Pujade-Villar, J. 2006. Revision of the genus Prosaspicera Kieffer, 1907 (Hym.: Figitidae: Aspicerinae). Zootaxa, 1379, 1-102.

Ros-Farré, P. \& Pujade-Villar, J. 2007. Plectocynipinae, a new subfamily of Figitidae and description of a new Neotropical genus of Thrasorinae (Hymenoptera: Cynipoidea). Zootaxa, 1583, $1-13$.

Ros-Farré, P., Ronquist, F. \& Pujade-Villar, J. 2000. Redescription of Acanthaegilips Ashmead, 1897, with characterization of the Anacharitinae and Aspiceratinae (Hymenoptera: Cynipoidea: Figitidae). Zoological Journal of the Linnean Society, 129: 467488.

SIAP. 2013. Servicio de Información Agroalimentaria y Pesquera. Ciclo: cíclicos-perennes 2013. http://www.siap.gob.mx/cierre-de-laproduccion-agricola-por-estado/. (Consulta 15-03-2014).

Vanegas-Rico, J. M., Lomeli-Flores, J. R., Rodríguez-Leyva, E., Mora-Aguilera, G. \& Valdez, J. M. 2010. Enemigos naturales de Dactylopius opuntiae (Cockerell) en Opuntia ficus-indica (L.) Miller en el centro de México. Acta Zoológica Mexicana (n.s.), 26: 415-433.

Weld, L. H. 1952. Cynipoidea (Hym.) 1905-1950 is being a Supplement to the Dalla Torre and Kieffer monograph, the Cynipidae in Das Tierreich, Leiferung 24, 1910 and bringing the systematic literature of the world up to date, including keys to families and subfamilies and list of new generic, specific and variety names. Ann Arbor, Michigan, Privately Printed: 351 pp. 